Fritsche, Nicole; Hirsch, Julia

\title{
Marketing im Gastgewerbe. Von der Fachwissenschaft zum beruflichen Lehramtsstudium
}

Haushalt in Bildung \& Forschung 5 (2016) 1, S. 60-75

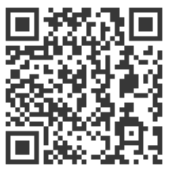

Quellenangabe/ Reference:

Fritsche, Nicole; Hirsch, Julia: Marketing im Gastgewerbe. Von der Fachwissenschaft zum beruflichen Lehramtsstudium - In: Haushalt in Bildung \& Forschung 5 (2016) 1, S. 60-75 - URN:

urn:nbn:de:0111-pedocs-202763 - DOI: 10.25656/01:20276

https://nbn-resolving.org/urn:nbn:de:0111-pedocs-202763

https://doi.org/10.25656/01:20276

in Kooperation mit / in cooperation with:

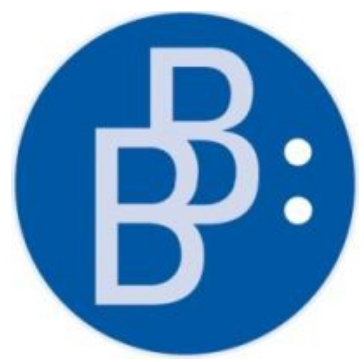

https://www.budrich.de

\section{Nutzungsbedingungen}

Gewährt wird ein nicht exklusives, nicht übertragbares, persönliches und beschränktes Recht auf Nutzung dieses Dokuments. Dieses Dokument ist ausschließlich für den persönlichen, nicht-kommerziellen Gebrauch bestimmt. Die Nutzung stellt keine Übertragung des Eigentumsrechts an diesem Dokument dar und gilt vorbehaltlich der folgenden Einschränkungen: Auf sämtlichen Kopien dieses Dokuments müssen alle Urheberrechtshinweise und sonstigen Hinweise auf gesetzlichen Schutz beibehalten werden. Sie dürfen dieses Dokument nicht in irgendeiner Weise abändern, noch dürfen Sie dieses Dokument für öffentliche oder kommerzielle Zwecke vervielfältigen, öffentlich ausstellen, aufführen, vertreiben oder anderweitig nutzen. Mit der Verwendung dieses Dokuments erkennen Sie die

\section{Terms of use}

We grant a non-exclusive, non-transferable, individual and limited right to using this document.

This document is solely intended for your personal, non-commercial use. Use of this document does not include any transfer of property rights and it is conditional to the following limitations: All of the copies of this documents must retain all copyright information and other information regarding legal protection. You are not allowed to alter this document in any way, to copy it for public or commercial purposes, to exhibit the document in public, to perform, distribute or otherwise use the document in public.

By using this particular document, you accept the above-stated conditions of use.

\section{Kontakt / Contact:}

\section{peDOcs}

DIPF | Leibniz-Institut für Bildungsforschung und Bildungsinformation Informationszentrum (IZ) Bildung

E-Mail:pedocs@dipf.de

Internet: www.pedocs.de

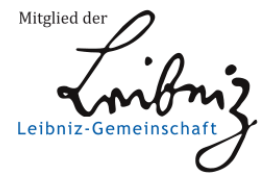


5. Jahrgang Heft 1 2016

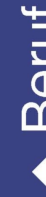

$\frac{1}{5}$

$\diamond$

(1)

(1)

ชิ

此

$\varepsilon$

ธิ

을

$\stackrel{\overline{1}}{>}$

$\bullet$

(1)

8

$\frac{1}{c}$

ब

$\varepsilon$

ह

क

N

$\bullet$

$\pm$

$\frac{1}{3}$
$\frac{3}{5}$
$+\frac{1}{0}$
$\frac{5}{0}$
$\frac{5}{5}$
0
0
0

ISSN 2193-8806

\section{Haushalt in}

Berufliche Bildung

Gestaltungsmöglichkeiten und

Zukunftsoptionen

IONDEILINL

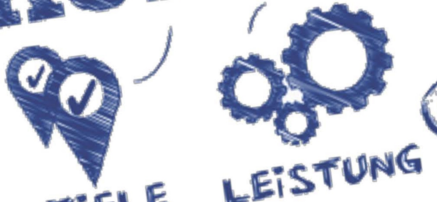

ZiEle leistung

FAHIGKEETEN
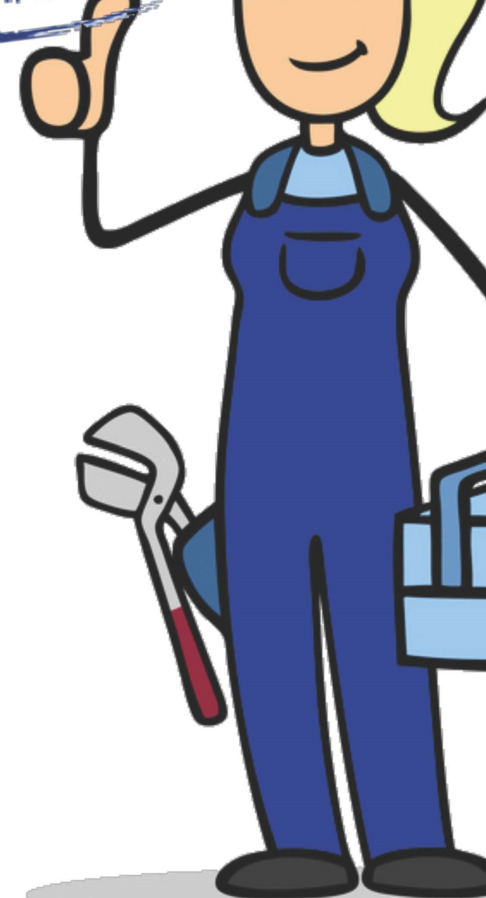
Inhaltsverzeichnis |

Julia Kastrup \& Irmhild Kettschau

Editorial.

Julia Kastrup \& Irmhild Kettschau

Standortentwicklungen, Nachwuchssituation und Nachwuchsförderung

in der beruflichen Fachdidaktik

Michael Martin

Der Berufsdidaktische Dreidecker.

Christine Küster, Nadine Harle \& Malte Matthias Flöper

Heterogenität als Zukunftsoption: Gestaltungsmöglichkeiten

des individualisierten Unterrichts in der beruflichen Bildung.

Anna Hoff

Interkulturelle Kompetenzen - Ein Blick auf die berufliche

Handlungsfähigkeit.

Julia Hirsch \& Nicole Fritsche

Marketing im Gastgewerbe - Von der Fachwissenschaft

zum beruflichen Lehramtsstudium

Julia Kastrup \& Gorden Steinke

Essbiografisches Lernen mit minderjährigen Flüchtlingen

in der Berufsorientierung.

Meike Ernestine Tecklenburg

Nachhaltiges Handeln in der Kita-Verpflegung:

Herausforderungen für die Weiterbildung.....

Marie Nölle

Nachhaltigkeitsbezogene Weiterbildungen im Berufsfeld

Ernährung und Gesundheit - ein Überblick zu Angebot und Bedarf 
Marketing im Gastgewerbe

Nicole Fritsche \& Julia Hirsch

\section{Marketing im Gastgewerbe - Von der Fachwissenschaft zum beruflichen Lehramtsstudium}

Das berufliche Lehramtsstudium der Universität Paderborn in Kooperation mit der Hochschulschule Ostwestfalen-Lippe beinhaltet neben der Fachdidaktik und den Bildungswissenschaften Module der Fachwissenschaften. Der Artikel vergleicht Inhalte des Seminars "Grundlagen des Marketings" mit den Lehrplänen des Gastgewerbes und verdeutlicht den Stellenwert der Fachwissenschaft für das zukünftige berufliche Handlungsfeld der Studierenden. Abschließend werden Möglichkeiten der didaktischen Umsetzung an der Hochschule skizziert.

Schlüsselwörter: Berufliche Lehrerbildung, Fachwissenschaft, Handlungsorientierung, Gastgewerbe, Marketing

\section{Berufliches Lehramt im Berufsfeld Ernährung \& Hauswirtschaft}

\subsection{Beruflicher Lehramtsstudiengang der Universität Paderborn in Kooperation mit der Hochschule Ostwestfalen-Lippe}

Die Universität Paderborn bietet gemeinsam mit der Hochschule Ostwestfalen-Lippe seit dem Wintersemester 2012/2013 den Studiengang Lehramt an Berufskollegs mit den zwei beruflichen Fachrichtungen Ernährungs- und Hauswirtschaftswissenschaft sowie Lebensmitteltechnik an.

Der Studiengang umfasst eine sechssemestrige Bachelor- sowie eine viersemestrige Masterphase. Beide beinhalten ein bildungswissenschaftliches und berufspädagogisches Studium, das Studium beider beruflicher Fachrichtungen sowie Praxiselemente. Das Studium der beruflichen Fachrichtungen setzt sich dabei aus fachwissenschaftlichen und fachdidaktischen Anteilen zusammen. Darüber hinaus ist im Bachelorstudiengang das Modul „,Deutsch für Schülerinnen und Schüler mit Zuwanderungsgeschichte“ (DaZ) verpflichtend (Universität Paderborn \& Hochschule Ostwestfalen-Lippe, 2015). Der Bachelorstudiengang hat insgesamt einen Umfang von 180 Leistungspunkten (LP) und wird mit dem akademischen Grad „Bachelor of Education“ abgeschlossen. Der Masterstudiengang weist einen Umfang von 120 Leistungspunkten (LP) auf und führt zum akademischen Grad ,Master of Education". 
Marketing im Gastgewerbe |

\subsection{Ablauf des beruflichen Lehramtsstudiums}

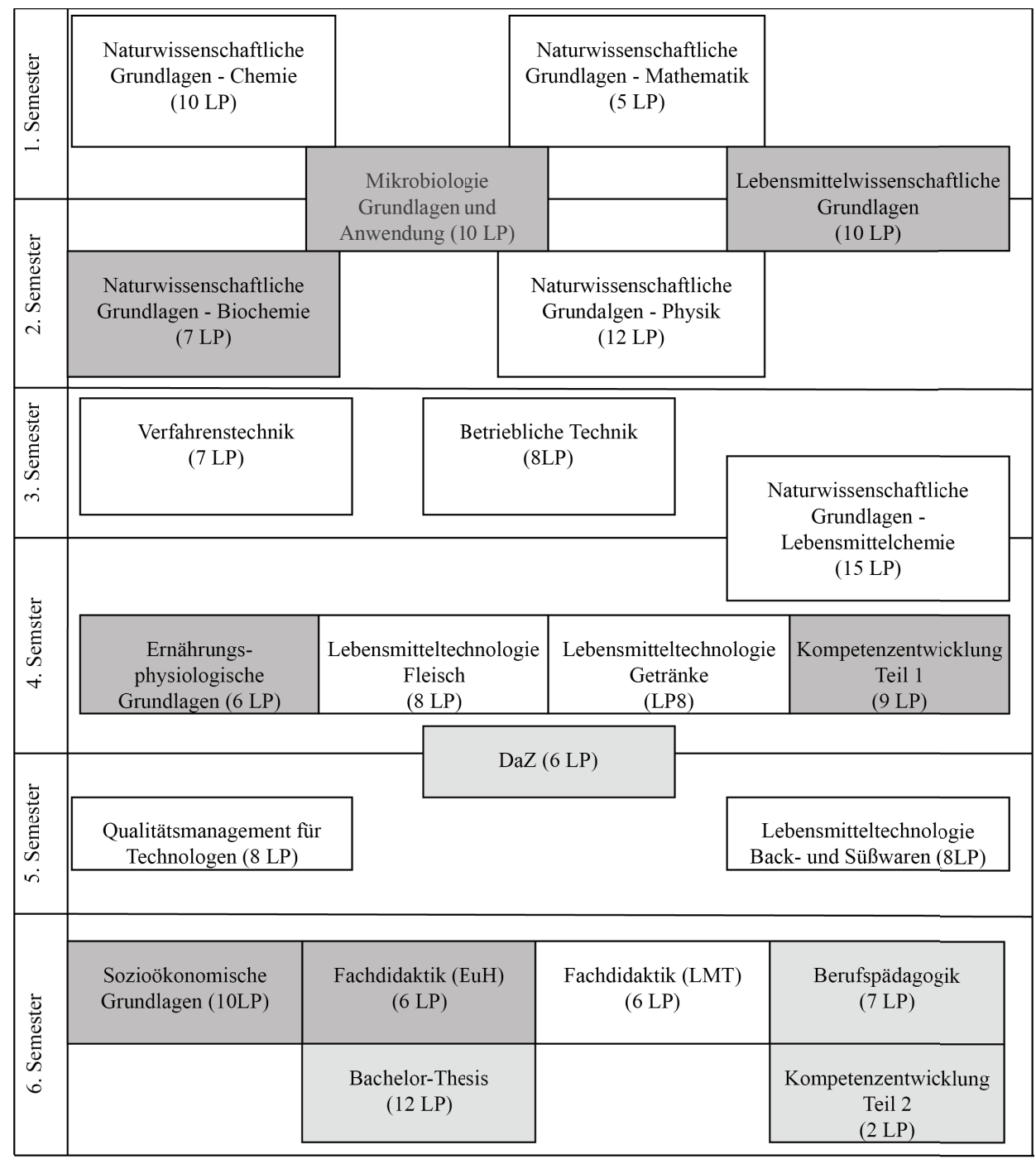

Ernährungs- und Hauswirtschaftswissenschaft (59 LP)
Lebensmitteltechnik (85 LP)
Bildungswissenschaften (18 LP)

Abb. 1: Studienverlaufsplan des Bachelorstudiengangs Lehramt an Berufskollegs (Quelle: eigene Darstellung nach Universität Paderborn \& Hochschule Ostwestfalen-Lippe, 2015) 


\section{Marketing im Gastgewerbe}

Abbildung 1 zeigt einen Überblick über den Studienverlauf des BachelorStudiengangs mit den Modulen beider beruflicher Fachrichtungen sowie des bildungswissenschaftlichen und berufspädagogischen Studiums. Die Module des Masterstudiengangs sind für den vorliegenden Artikel irrelevant und werden daher nicht näher betrachtet.

Das Studium des Lehramts an Berufskollegs mit den beruflichen Fachrichtungen Ernährungs- und Hauswirtschaftswissenschaft sowie Lebensmitteltechnik ist modularisiert. Die Module bestehen in der Regel aus mehreren Lehrveranstaltungen, die inhaltlich aufeinander abgestimmt sind.

In den ersten fünf Bachelorsemestern lernen die Studierenden an der Hochschule Ostwestfalen-Lippe die naturwissenschaftlichen und technischen Grundlagen für beide berufliche Fachrichtungen. Im Anschluss wechseln sie an die Universität Paderborn und vertiefen hier im sechsten Bachelorsemester sowie im Masterstudium ihre Kompetenzen in den Ernährungs- und Hauswirtschaftswissenschaften, der Fachdidaktik, den Bildungswissenschaften und der Berufspädagogik.

\subsection{Handlungsfelder der Lehrkräfte am Berufskolleg}

Die schulischen Handlungsfelder der Lehrkräfte mit den zwei beruflichen Fachrichtungen Ernährungs- und Hauswirtschaftswissenschaft sowie Lebensmitteltechnik sind aufgrund der Polyfunktionalität beruflicher Schulen im Allgemeinen und der Heterogenität des korrespondierenden Berufsfeldes Ernährung und Hauswirtschaft im Speziellen sehr vielfältig.

Das Berufsfeld Ernährung und Hauswirtschaft vereint knapp 30 Berufe, deren zugehörige Bildungsgänge potentielle Einsatzgebiete der zukünftigen Lehrkräfte sind (Kettschau, 2013, S. 3). Das Spektrum reicht dabei von gewerblich-technischen bzw. handwerklich ausgerichteten Berufen der Ernährungs- und Lebensmittelbranche über branchenspezifische Verkaufsberufe sowie Berufe des Hotel- und Gastgewerbes bis zu weiteren personenbezogenen Dienstleistungsberufen wie z. B. die Berufe der Hauswirtschaft (ebd.). Der Schwerpunkt des Berufsfeldes Ernährung und Hauswirtschaft liegt auf den Berufen, die im dualen System nach Berufsbildungsgesetz (BBiG) oder der Handwerksordnung (HwO) ausgebildet werden (ebd. S. 4). Ihm gehören 20 der knapp 30 Berufe an. Dazu zählen beispielsweise die Ausbildungsberufe Bäckerin/Bäcker, Fleischerin/Fleischer, Fachverkäuferin/Fachverkäufer im Lebensmittelhandwerk, Köchin/Koch, Restaurantfachfrau/Restaurantfachmann, Fachfrau/Fachmann für Systemgastronomie, Fachkraft für Lebensmitteltechnik sowie Hauswirtschafterin/Hauswirtschafter. Hinzu kommen acht Berufe, die außerhalb BBiG/HwO im vollzeitschulischen Bereich der Berufsfachschulen nach Landesrecht ausgebildet werden (Friese, 2010, S. 319). Ein Beispiel hierfür ist die/der staatlich geprüfte Assistentin/staatlich geprüfte Assistent für Ernährung und Versorgung mit dem Schwerpunkt Service. Ferner können die zukünftigen Lehrkräfte in Bildungsgängen unterrichten, die allgemeinbildende Abschlüsse der Sekundarstufe II 
vermitteln (z. B. Fachoberschule und Berufliches Gymnasium) oder der Ausbildungsvorbereitung sowie der beruflichen Weiterbildung dienen (Fachschule). Potenzielle Einsatzfelder für zukünftige Lehrkräfte der beruflichen Fachrichtung Ernährung und Hauswirtschaft sind darüber hinaus Berufe bzw. Bildungsgänge mit Bezügen zu ernährungs- und hauswirtschaftswissenschaftlichen Themen, die aber nicht primär zum Berufsfeld Ernährung und Hauswirtschaft zählen. Hierzu zählen beispielsweise die/der staatlich geprüfte Kinderpflegerin/der staatlich geprüfte Kinderpfleger oder die/der staatlich anerkannte Erzieherin/staatlich anerkannte Erzieher.

Lehrkräfte mit den zwei beruflichen Fachrichtungen Ernährungs- und Hauswirtschaftswissenschaft sowie Lebensmitteltechnik sind insgesamt also mit einem sehr heterogenen Handlungsfeld konfrontiert. Sie ,müssen über umfangreiche Qualifikationen verfügen und ein hohes $\mathrm{Ma} \beta$ an Flexibilität, aber auch Neugier und Anpassungsbereitschaft mitbringen" (Stomporowski, 2011, S. 9).

\section{Vergleich der Inhalte der Lehrpläne des Gastgewerbes mit den Inhalten des Moduls Sozioökonomische Grundlagen}

\subsection{Struktur der Berufsausbildung im Gastgewerbe}

Im Jahr 2013 befanden sich deutschlandweit insgesamt 112134 Auszubildende in einem der 20 Ausbildungsberufe des Berufsfeldes Ernährung und Hauswirtschaft nach $\mathrm{BBiG} / \mathrm{HwO}$. Die Berufe des Gastgewerbes nehmen dabei gemessen an der Anzahl der Auszubildenden (Stichtag 31.12.2013) den größten Stellenwert innerhalb des dualen Systems des Berufsfeldes Ernährung und Hauswirtschaft ein. Über 67000 Auszubildende lernten 2013 einen der sechs gastgewerblichen Berufe. Folglich wurden $60 \%$ der Ausbildungsverträge des Berufsfeldes Ernährung und Hauswirtschaft im Berufsbereich des Gastgewerbes geschlossen (s. Tabelle 1). Aufgrund dieser zentralen Stellung wird im weiteren Verlauf des Artikels exemplarisch der Berufsbereich des Gastgewerbes betrachtet (Bundesinstitut für Berufsbildung (BiBB), 2015, Kettschau \& Wirth, 2015).

$\mathrm{Zu}$ den Ausbildungsberufen im Gastgewerbe zählen laut dem Deutschen Hotelund Gaststättenverband (2015) folgende sechs Berufe:

- Köchin und Koch

- Fachkraft im Gastgewerbe

- Hotelfachfrau und Hotelfachmann

- Restaurantfachfrau und Restaurantfachmann

- Fachfrau und Fachmann für Systemgastronomie

- Hotelkauffrau und Hotelkaufmann 


\section{Marketing im Gastgewerbe}

Tab. 1: Daten ausgewählter Ausbildungsberufe nach BBiG/HwO im Berufsfeld Ernährung und Versorgung (Quelle: eigene Darstellung nach BiBB, 2015, Kettschau \& Wirth, 2015)

\begin{tabular}{|c|c|c|c|}
\hline Berufsbezeichnung & $\begin{array}{l}\text { Anzahl Auszu- } \\
\text { bildende am } \\
31.12 .2013 \\
\end{array}$ & $\begin{array}{l}\text { Summe Anzahl } \\
\text { Auszubildende } \\
2013 \\
\end{array}$ & $\begin{array}{l}\text { Anteil } \\
\text { in } \%\end{array}$ \\
\hline \multicolumn{4}{|c|}{ Berufe des Gastgewerbes } \\
\hline Hotelfachfrau/-mann & 22647 & \multirow{6}{*}{67305} & \multirow{6}{*}{60,02} \\
\hline Restaurantfachfrau/-mann & 7422 & & \\
\hline Köchin/Koch & 22890 & & \\
\hline Fachfrau/-mann für Systemgastronomie & 4641 & & \\
\hline Fachkraft im Gastgewerbe & 3975 & & \\
\hline Hotelkauffrau/-mann & 1089 & & \\
\hline \multicolumn{4}{|c|}{ Berufe des Nahrungsmittelhandwerks/der Lebensmittelherstellung } \\
\hline Bäckerin/Bäcker & 7659 & \multirow{10}{*}{20619} & \multirow{10}{*}{18,39} \\
\hline Konditorin/Konditor & 4248 & & \\
\hline Fleischerin/Fleischer & 4026 & & \\
\hline $\begin{array}{l}\text { Müllerin/Müller (Verfahrenstechnologin/ } \\
\text { Verfahrenstechnologe Mühlen und Futter- } \\
\text { mittelwirtschaft) }\end{array}$ & 267 & & \\
\hline Fachkraft für Süßwarentechnik & 270 & & \\
\hline Milchtechnologin/Milchtechnologe & 663 & & \\
\hline Fachkraft für Fruchtsafttechnik & 117 & & \\
\hline Brauerin/Brauer und Mälzerin/Mälzer & 837 & & \\
\hline $\begin{array}{l}\text { Speiseeisherstellerin/Speiseeishersteller } \\
\text { (Fachkraft für Speiseeis) }\end{array}$ & 39 & & \\
\hline Fachkraft für Lebensmitteltechnik & 2493 & & \\
\hline \multicolumn{4}{|c|}{ Personenbezogene Berufe } \\
\hline Hauswirtschafterin/Hauswirtschafter & 3579 & 3579 & 3,19 \\
\hline \multicolumn{4}{|c|}{ Berufe des Lebensmittelhandels/-verkauf } \\
\hline $\begin{array}{l}\text { Fachverkäuferin/Fachverkäufer im Lebens- } \\
\text { mittelhandwerk Schwerpunkt Konditorei }\end{array}$ & 939 & \multirow{3}{*}{20631} & \multirow{3}{*}{18,40} \\
\hline $\begin{array}{l}\text { Fachverkäuferin/Fachverkäufer im Lebens- } \\
\text { mittelhandwerk Schwerpunkt Bäckerei }\end{array}$ & 14589 & & \\
\hline $\begin{array}{l}\text { Fachverkäuferin/Fachverkäufer im Lebens- } \\
\text { mittelhandwerk Schwerpunkt Fleischerei }\end{array}$ & 5103 & & \\
\hline Gesamt & & 112134 & 100,00 \\
\hline
\end{tabular}




\section{Marketing im Gastgewerbe |}

Die Ausbildung erfolgt im dualen System, d. h. an den beiden Lernorten Betrieb und Berufsschule. Der schulische Teil der Ausbildung wird in NordrheinWestfalen (NRW) durch landeseigene Lehrpläne geregelt. Diese basieren auf den Rahmenlehrplänen der Ständigen Konferenz der Kultusminister der Länder (KMK) und beschreiben Ziele und Inhalte des berufsbezogenen Unterrichts.

Kennzeichnend für die Ausbildung im Gastgewerbe ist die Strukturierung nach dem grund- und fachberuflichen Ausbildungskonzept (Schelten, 2010, S. 97). Demzufolge findet im ersten Ausbildungsjahr für alle sechs gastgewerblichen Berufe eine gemeinsame berufliche Grundbildung auf Berufsfeldbreite statt. Im zweiten Ausbildungsjahr folgt die erste Stufe der beruflichen Fachbildung. Hier setzt sich die gemeinsame Ausbildung für die Berufe Fachkraft im Gastgewerbe, Hotelfachfrau/Hotelfachmann, Restaurantfachfrau/Restaurantfachmann, Fachfrau/Fachmann für Systemgastronomie weiter fort. Ausnahme stellt der Beruf der Köchin/des Koches dar. Für sie bzw. ihn beginnt im zweiten Ausbildungsjahr bereits eine besondere berufliche Fachbildung. Für die Berufe Hotelfachfrau/Hotelfachmann, Restaurantfachfrau/Restaurantfachmann und Fachfrau/Fachmann für Systemgastronomie erfolgt die Differenzierung durch die besondere berufliche Fachbildung erst im dritten Ausbildungsjahr. Die Ausbildung zur Fachkraft im Gastgewerbe endet bereits nach der ersten Stufe der beruflichen Fachbildung, d. h. mit dem zweiten Ausbildungsjahr. Im Anschluss besteht allerdings die Möglichkeit die Ausbildung in einem der anderen vier gastgewerblichen Berufe fortzusetzen, um die besondere berufliche Fachbildung zu ergänzen (Ministerium für Schule, Jugend und Kinder des Landes Nordrhein-Westfalen, 2014a, S. 32). Abbildung 2 verdeutlicht die Struktur des schulischen Teils der gastgewerblichen Ausbildung, gleichzeitig gibt sie einen Überblick über die Lernfelder.

Aus den Bezeichnungen der Lernfelder wird deutlich, dass eine korrespondierende Fachwissenschaft nicht klar zu benennen ist. Die Lernfelder verweisen auf ein breites Feld an Themen und damit verbunden auf eine Vielzahl von Wissenschaften. Diese reichen beispielweise von der Ernährungsphysiologie und der Hauswirtschaftswissenschaft über die Lebensmitteltechnologie bis hin zur Lebensmittelhygiene und dem Lebensmittelrecht. Darüber hinaus bestehen Bezüge zur Kommunikationswissenschaft, der Wirtschaftslehre und dem Marketing. Daher ist fachwissenschaftlich ein interdisziplinärer und auch multidisziplinärer Zugang in der Lehrkräfteausbildung erforderlich (Fegebank, 2010, S. 586). Im folgenden Kapitel wird das Modul Sozioökonomische Grundlagen und daraus exemplarisch das Seminar Grundlagen des Marketings vorgestellt, um im weiteren Verlauf des Artikels inhaltliche Bezüge zum schulischen Handlungsfeld des Gastgewerbes aufzuzeigen. 


\section{Marketing im Gastgewerbe}

Abb. 2: Ablauf und Struktur der schulischen Ausbildung der gastgewerblichen Berufe in NRW (Quelle: eigene Darstellung nach Ministerium für Schule, Jugend und Kinder des Landes Nordrhein-Westfalen, 2004a \& 2004b)

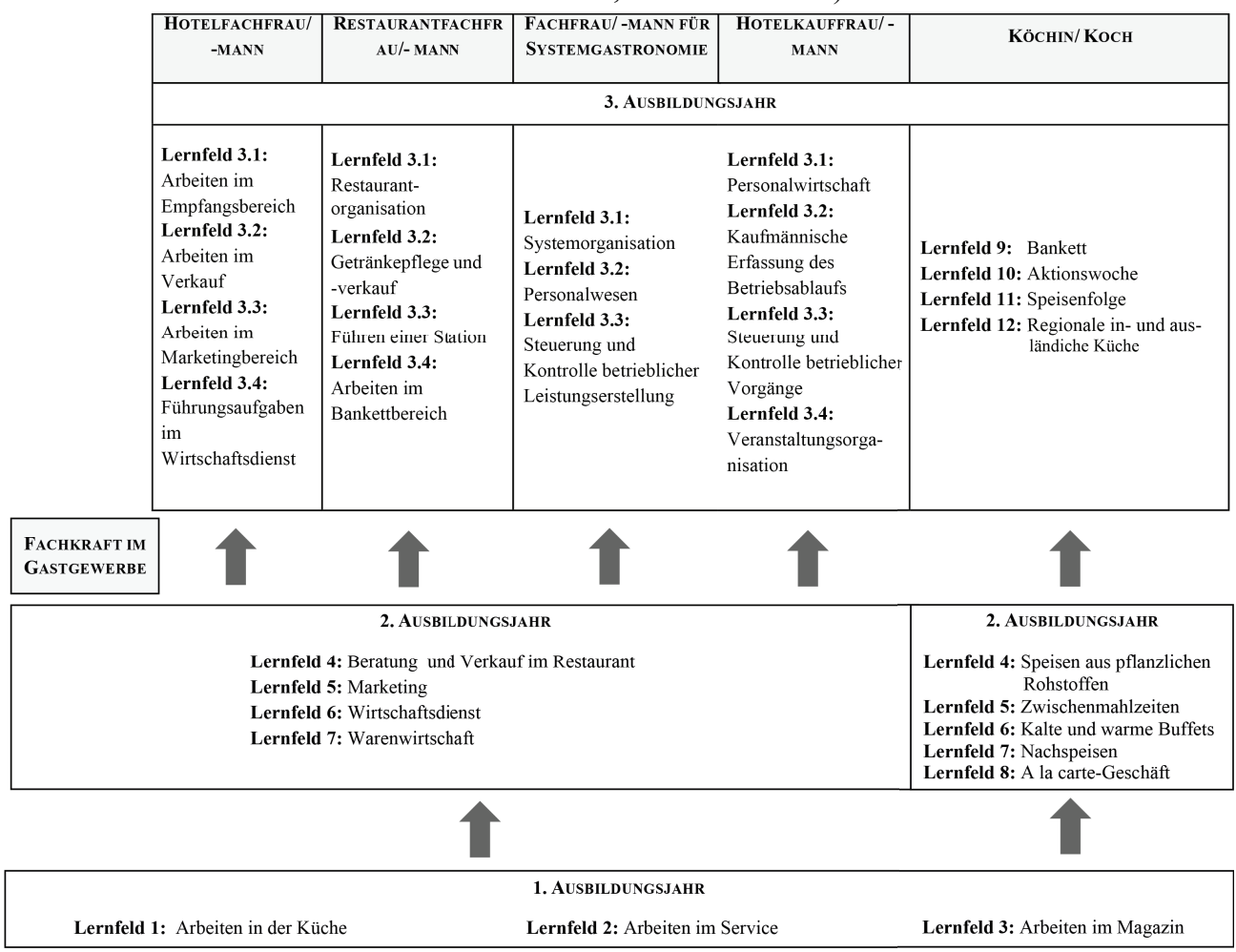

\subsection{Darstellung und Erläuterung der Inhalte des Seminars Grundlagen des Marketings}

Das Modul „Sozioökonomische Grundlagen“ ist für das sechste Semester des Bachelorstudiums vorgesehen und das erste fachwissenschaftliche Modul, welches die Studierenden an der Universität Paderborn absolvieren. Innerhalb des Moduls belegen die Studierenden parallel folgende drei Seminare:

- Wirtschaftslehre des Großhaushalts (4 LP)

- Individuum und Gesellschaft (3 LP)

- Grundlagen des Marketings (3 LP)

(Universität Paderborn \& Hochschule Ostwestfalen-Lippe, 2015) 


\section{Marketing im Gastgewerbe |}

Wie bereits erläutert, stammen die Inhalte der Lernfelder des Gastgewerbes aus verschiedenen Fachdisziplinen. Nichtsdestotrotz findet sich bei allen gastgewerblichen Berufen eine Gemeinsamkeit: Sie werden, wie alle Berufe der beruflichen Fachrichtung Ernährung und Hauswirtschaft, unter dem Aspekt ihrer wichtigsten beruflichen Arbeitsaufgabe den personenorientierten Fachrichtungen zugeordnet (Bader, Schröder \& Gebert, 2010, S. 216).

Bedürfnisse von Personen sind Ausgangspunkt wirtschaftlichen Handelns (Jung, 2010). Alle Berufe des Gastgewerbes sind durch regelmäßigen Kontakt mit Gästen gekennzeichnet, so dass bei Angebot und Absatz von Produkten sowie dem Erbringen einer Dienstleistung direkt auf Bedürfnisse und Verhaltensweisen der Gäste reagiert werden muss und Leistungen gegebenenfalls (auch spontan) einer Veränderung, bzw. einer entsprechenden Anpassung an die Wünsche der Kunden bedürfen.

Die Analyse der Bedürfnisse der aktuellen und potentiellen Kundengruppen bzw. Gäste sollte dabei Ausgangspunkt aller Überlegungen eines Unternehmens sein. Sie ist dem Marketing zuzuordnen (Meffert, Burmann \& Kirchgeorg, 2015, S. 9f.). Marketing ist dabei eine gleichberechtigte Unternehmensfunktion, d. h. dass Entscheidungen aller Abteilungen und somit die Führung des gesamten Unternehmens am Markt orientiert sind und mit den Unternehmenszielen übereinstimmen (Meffert et al., 2010, S. 14). Wissenschaftlich ist Marketing der Betriebswirtschaftslehre zuzuordnen (Jung, 2010, S. 551).

Die konstitutiven Besonderheiten von Dienstleistungen führen dazu, dass die klassischen Marketingansätze nicht einfach auf die der Dienstleistungen übertragen werden können, sondern ein eigenständiger Ansatz des Dienstleistungsmarketing entwickelt wurde (Meffert et al., 2010, S. 28). Aufgrund dessen sind im vorgestellten Lehramtsstudiengang im gesamten Verlauf des Seminars die klassischen Marketingansätze sowie immer auch die besonderen Überlegungen im Bereich der Dienstleistungen relevant.

Elementar für das Seminar sind die allgemeinen Ziele und Aufgaben des Marketings in einem Unternehmen. Die besonderen Eigenschaften von Dienstleitungen bringen wiederum Implikationen für das Marketing in Dienstleistungsbetrieben und somit auch im Gastgewerbe mit sich (Meffert \& Bruhn, 2006, S. 63). Diese finden sich ebenfalls in den Inhalten des Seminars wieder.

Die Markt- und Kundensegmentierung hat eine besondere Bedeutung für betriebliche Entscheidungen, da die Überlegungen, welche Personengruppen mit Angeboten erreicht werden sollen, die Grundlage für alle weiteren Überlegungen, Handlungen und Strategien bilden. Den Ausgangspunkt für die Segmentierung bildet die Analyse des Käuferverhaltens, die wiederum Grundvoraussetzung für die Durchführung jeglicher Marketingaktivitäten ist (Meffert \& Bruhn, 2006, S. 115). Die Studierenden sollen bei diesen Themen u. a. eine Vorstellung davon erhalten, welche Einflussfaktoren den Kaufentscheidungsprozess bedingen und bei der Festlegung der Marketingstrategien eine Rolle spielen. Diese Kenntnisse führen zur Systematisierung der 


\section{Marketing im Gastgewerbe}

Marketinginstrumente in die (im klassischen Gütermarketing vorliegenden) vier Mixbereiche der Produkt-, Kommunikations-, Distributions- sowie der Preispolitik. Im Bereich des Dienstleistungsmarketings werden diese um den Bereich der Personalpolitik ergänzt (Meffert \& Bruhn, 2006, S. 389). Dieser ist dem internen Marketing zuzuordnen und beinhaltet alle personalbezogenen Aspekte, die bei der Erstellung und dem Angebot von Dienstleistungen aus Marketingsicht berücksichtigt werden müssen. Hintergrund dieses eigenen internen Bereichs ist die, durch den permanenten Kundenkontakt bestehende, Notwendigkeit von fähigem, geschultem und kundenorientiertem Personal in der gesamten Dienstleistungs-branche (ebd., S. $623)$.

Relevant für die Studierenden sind vor allem die Merkmale, Aufgaben, Instrumente und Möglichkeiten der Mixbereiche für das klassische Konsumgütermarketing sowie für das Dienstleistungsmarketing. Die kommunikativen Marketinginstrumente des Gastgewerbes spielen dabei im Seminar eine besondere Rolle, da diese grundlegend für den Erfolg von Unternehmen dieser Branche sind.

\subsection{Vergleich der Lehrpläne mit den Inhalten des Seminars}

Im Folgenden werden die Inhalte der aktuell gültigen Lehrpläne für das Berufskolleg in NRW für die Ausbildungsberufe im Gastgewerbe mit denen des Seminars Grundlagen des Marketings an der Universität Paderborn verglichen. Ziel ist aufzuzeigen, inwieweit die Inhalte der Fachwissenschaft der Universität für das spätere Handlungsfeld an beruflichen Schulen bedeutsam sind. Dies wird exemplarisch für das Themengebiet Marketing diskutiert. Berücksichtigt werden dabei die Inhalte der Lernfelder der Lehrpläne der sechs dualen Ausbildungsberufe des Gastgewerbes in NRW.

Für den Vergleich wurden im ersten Schritt die Lehrpläne des Gastgewerbes gesichtet, um dabei die Lernfelder zu identifizieren, die grundsätzlich einen Bezug zum Marketing aufweisen. Im nächsten Schritt wurden die Inhalte der identifizierten Lernfelder konkret mit den Inhalten des Seminars Grundlagen des Marketings verglichen, um herauszuarbeiten, an welcher Stelle Schnittmengen und Differenzen vorzufinden sind. Tabelle 2 zeigt die Ergebnisse dieses Abgleichs. Dabei wurden zur besseren Übersicht nur die Inhalte der Lernfelder und des Seminars aufgeführt, die Parallelen aufweisen.

Der Abgleich der Inhalte zeigt auf der einen Seite, dass sich fast alle Inhalte des Seminars an der Universität Paderborn in den Lernfeldern der gastgewerblichen Berufe wiederfinden. Auf der anderen Seite lässt sich kein Lernfeld ermitteln, das Inhalte behandelt, die nicht im Seminar thematisiert werden. 


\section{Marketing im Gastgewerbe |}

Tab. 2: Abgleich der Inhalte der Lernfelder des Gastgewerbes mit denen des Seminars Grundlagen des Marketings (Quelle: eigene Darstellung in Anlehnung an Ministerium für Schule, Jugend und Kinder des Landes Nordrhein-Westfalen, 2004a \& 2004b)

\begin{tabular}{|c|c|}
\hline $\begin{array}{l}\text { Inhalte der Lernfelder der gastgewerb- } \\
\text { lichen Berufe }\end{array}$ & $\begin{array}{l}\text { Inhalte des Seminars Grundlagen des } \\
\text { Marketings }\end{array}$ \\
\hline \multicolumn{2}{|l|}{ 2. Ausbildungsjahr } \\
\hline $\begin{array}{l}\text { Lernfeld 2.2 Marketing } \\
\text { (gastgewerbliche Berufe) } \\
\text { - Ziele und Aufgaben des Marketings } \\
\text { - } \quad \text { Marketinginstrumente und Marke- } \\
\text { - tingmix } \\
\text { - } \quad \text { Verkaufsförderung } \\
\quad \text { Werbemittel }\end{array}$ & $\begin{array}{l}\text { - } \text { Aufgaben und Entwicklungslinien des } \\
\text { Marketings } \\
\text { - } \quad \text { Marketinginstrumente/-mix } \\
\text { - Marketinginstrumente bei } \\
\text { - } \quad \text { Kienstleistungsbetrieben } \\
\text { Kommunikationsinstrumente }\end{array}$ \\
\hline \multicolumn{2}{|l|}{ 3. Ausbildungsjahr } \\
\hline $\begin{array}{l}\text { Lernfeld 3.3 Arbeiten im Marketingbe- } \\
\text { reich (Hotelfachfrau/-mann) } \\
\text { - Marketingmaßnahmen }\end{array}$ & $\begin{array}{l}\text { - Beispiele für den Einsatz der } \\
\text { Marketinginstrumente }\end{array}$ \\
\hline $\begin{array}{l}\text { Lernfeld 3.4 Führungsaufgaben im } \\
\text { Wirtschaftsdienst (Hotelfachfrau/- } \\
\text { mann) } \\
\text { - Innerbetriebliche Kommunikation } \\
\text { - } \quad \text { Bedeutung von Motivation, Führungs- } \\
\quad \text { stil und Training } \\
\end{array}$ & - $\quad$ Personalpolitik \\
\hline $\begin{array}{l}\text { Lernfeld 3.1 Systemorganisation (Fach- } \\
\text { frau/-mann für Systemgastronomie) } \\
\text { - Merkmale standardisierter Konzepte } \\
\text { - } \quad \text { Marketing }\end{array}$ & - Teile der Distributionspolitik \\
\hline $\begin{array}{l}\text { Lernfeld 3.1 Personalwirtschaft } \\
\text { (Hotelkauffrau/-mann) } \\
\text { - Mitarbeiterführung, -betreuung und } \\
\quad \text {-schulung } \\
\text { - } \quad \text { Mitarbeiterbeurteilung } \\
\end{array}$ & $\begin{array}{ll}\text { - } & \text { Mitarbeiterkommunikation } \\
\text { - } & \text { Instrumente der Personalveränderung }\end{array}$ \\
\hline $\begin{array}{l}\text { Lernfeld 3.2 Aktionswoche } \\
\text { (Köchin/Koch) } \\
\text { - Werbung und Verkaufsförderung }\end{array}$ & - Kommunikationsinstrumente \\
\hline
\end{tabular}

Das Basiswissen des Marketings, also die Aufgaben und Ziele des Marketings, die Marketinginstrumente im Allgemeinen sowie die Kommunikationsinstrumente im Besonderen, sind in Lernfeld 2.2 Marketing der gemeinsamen Grundbildung der gastgewerblichen Berufe zu finden. Im dritten Ausbildungsjahr erfolgt eine Vertiefung in die Thematik des Marketings in allen Ausbildungsberufen außer bei der/dem 


\section{Marketing im Gastgewerbe}

zur/zum Restaurantfachfrau/-mann. Die meisten Themen des Marketings finden sich in der Ausbildung zur/zum Hotelfachfrau/-mann.

Zusammenfassend lässt sich festhalten, dass das Seminar alle Inhalte abdeckt, die laut Lehrplan für die Berufe des Gastgewerbes im Marketing relevant sind. Darüber hinaus werden im Seminar an der Universität Paderborn weitere Themenfelder des Marketings behandelt, die nicht in den Lehrplänen des Gastgewerbes zu finden sind. Hierzu zählen zum Beispiel die Marktsegmentierung und die Marktforschung. Notwendig sind sie dennoch, da sie zum Gesamtverständnis des Marketings wesentlich sind. Zudem geht das Handlungsfeld der zukünftigen Lehrkräfte am Berufskolleg über das des Gastgewerbes hinaus. Inhalte des Seminars (und evtl. auch noch darüber hinausgehende) sind daher dennoch von Bedeutung.

\section{Skizze eines didaktischen Konzepts für das Seminar Grundlagen des Marketings}

Nachfolgend wird die Skizze einer möglichen hochschuldidaktischen Umsetzung des Seminars aufgezeigt und diskutiert.

Die Bologna-Reform erfordert für die didaktische Konzeption der Lehre an Hochschulen ein Umdenken. Berufsfeldrelevante Abschlüsse benötigen eine stärkere Orientierung an den zu erwerbenden Kompetenzen, deren Erwerb sich in einer dynamischen Kombination aus Wissen, Fertigkeiten, Fähigkeiten und Einstellungen äußert (Ertel \& Wehr, 2007, S. 13). Darüber hinaus ist es das Ziel der akademischen Ausbildung, eine möglichst hohe kognitive Verarbeitung und ein Verständnis der Lehrinhalte bei den Lernenden zu erreichen, damit eine möglichst breite Anwendbarkeit und Handlungsfähigkeit in verschiedenen Situationen erreicht wird (Seidel \& Krapp, 2014, S. 448f.).

Ziel der Berufsschule ist laut KMK die Entwicklung von Handlungskompetenz auf Seiten der Auszubildenden. Handlungskompetenz „entfaltet sich [dabei] in den Dimensionen von Fachkompetenz, Humankompetenz und Sozialkompetenz“ (KMK, 2011, S. 15) und liefert den Rahmen für die Formulierung aller Ziele der beruflichen Bildung. Das Konzept des handlungsorientierten Unterrichts liefert dabei den didaktischen Grundsatz (ebd., S. 17). Handlungsorientierter Unterricht ist Lernen anhand einer vollständigen Handlung. Über die Lösung komplexer beruflicher Aufgaben, die in Form des direkten Handels erprobt werden, wird dabei die Theorie zur Lösung des Problems erarbeitet (Bader, 2004, S. 63).

Der handlungsorientierte Unterricht ist der Erkenntnistheorie des moderaten Konstruktivismus zuzuordnen (Schelten, 2010, S. 177ff.). Der Konstruktivismus vertritt u. a. die These, dass Lernen nicht das einfache passive Aufnehmen und Abspeichern von Informationen ist, sondern ein aktiver Prozess ist, in dem sich die Lernenden individuell und selbstgesteuert mit dem Lerngebiet auseinandersetzen. Lerngegenstände stehen dabei in einem konkreten Situationsbezug und der Umgang mit 


\section{Marketing im Gastgewerbe |}

diesen hängt stark von den Vorkenntnissen und Erfahrungen der Personen ab, die ihr Wissen selbst entwickeln und in ihre Wissensstruktur einbetten (Schelten, 2010). Lernende sollen darüber hinaus Strategien entwickeln, mit deren Hilfe sie Informationen selbstständig bewerten und auswählen können und die Fähigkeit und Motivation besitzen, das ganze Leben lang selbstständig zu lernen. Aus konstruktivistischer Sicht ebnen die Lehrpersonen den Lernenden den Weg, der zu einem tieferen Verstehen führt. Er oder sie unterstützt, berät und regt diesen Prozess an. Allerdings müssen die Lernenden diesen selber gehen. Die Aktivität liegt also bei ihnen (Mietzel, 2007, S. 42).

Für das in diesem Artikel diskutierte Seminar Grundlagen des Marketings ist die Methode der Projektarbeit in Form des problemorientierten Lernens eine Umsetzungsmöglichkeit des handlungsorientierten Unterrichts. Bei dieser Methode steht ein Problem im Fokus, für das Lösungsmöglichkeiten entwickelt werden müssen. Anhand der Auseinandersetzung mit der Thematik des Marketings an einem speziellen Fall werden die Inhalte von den Studierenden in Kleingruppenarbeit vertieft (Brinker \& Schumacher, 2014, S. 107). Das spezielle Problem kann dabei als konkrete Situation angesehen werden, die Grundlage für das handlungsorientierte Lernen ist (Gudjons, 2003, S. 103).

Erziehungswissenschaftler sind sich einig darüber, dass sich das Lernen in Projekten sehr gut dafür eignet, neben den fachlichen auch die überfachlichen Kompetenzen zu fördern (Kuhlmeier \& Uhe, 1998, S. 126). Die Studierenden machen auf diese Weise eigene Erfahrungen mit einer Methode des handlungsorientierten Unterrichts, nach dem Unterricht gestaltet werden sollte. Außerdem wecken Projekte im Lehramtsstudium die Hoffnung, dass die Studierenden später qualifiziert und motiviert sind, Projekte auch in den beruflichen Schulen mit den Auszubildenden durchzuführen (ebd., S. 126).

Die Studierenden arbeiten dabei im vorliegenden Seminar in Kleingruppen an fiktiven Fällen. Solche Fälle bzw. Probleme sind im Rahmen des Marketings beispielsweise die Einführung eines neuen Burgers in einem Gastronomiebetrieb.

Bevor die Studierenden in Kleingruppen an den konkreten Problemen arbeiten, wird an zwei Präsenztagen das Basiswissen gemeinsam von der Seminarleitung und den Studierenden erarbeitet. Dabei werden die grundlegenden Begrifflichkeiten geklärt sowie Aspekte der Marktsegmentierung und der Marketingstrategien allgemein erarbeitet. Dieses Wissen ist notwendig, um an den einzelnen Fällen arbeiten zu können und die Aspekte für den jeweiligen Fall zu konkretisieren und auszuarbeiten. Studien und Marktanalysen, die in der Praxis bereits durchgeführte Marketingstrategien und deren Erfolg darstellen, sollen die Ideen und Überlegungen der Projektgruppen untermauern. Die Studierenden bekommen einen Ablaufplan, der die Formulierung der Aufgabe mit allen Teilschritten enthält. Darüber hinaus werden Meilensteine sowie Termine für Zwischenpräsentationen festgelegt. An diesen gemeinsamen Sitzungen können bisherige Überlegungen und Ideen diskutiert werden. 


\section{Marketing im Gastgewerbe}

Des Weiteren können sich die Gruppen gegenseitig Rückmeldungen geben und eventuelle Schwachstellen oder neue Ideen aufzeigen. Die Sozialform Gruppenarbeit sowie das gegenseitige Vorstellen der Zwischenergebnisse der Gruppen begünstigt das gemeinschaftliche Lernen und fördert die Sozialkompetenz der Studierenden (Schelten, 2010, S. 179). Bei der Lernmethode der Projektarbeit steht nicht nur das Lernprodukt im Zentrum, sondern auch der gesamten Lernprozess. Daher ist es sinnvoll zur Bewertung und Beurteilung des gesamten Projektes nicht nur herkömmliche Prüfungsverfahren anzuwenden, sondern diese z. B. durch eine Reflexion, bei der individuelle Lernfortschritte und Verbesserungen der eigenen Lernstrategie beurteilt werden, zu ergänzen. Das Ganze sollte zum Ende des Projektes in Form eines Lernportfolios von jedem Studierenden abgegeben werden (ebd., S. 180).

Vorteile des projektorientierten Lernens sind $u$. a. die praktische und praxisnahe Arbeit sowie das kollaborative Lernen innerhalb der Projektgruppe und über diese hinaus. Studierende können sich mit ihren Kenntnissen gegenseitig ergänzen und vom Wissen der anderen lernen. Außerdem wird bei den Studierenden durch die Handlungsorientierung die Motivation gesteigert. Durch diese Art des Lernens erwerben die Studierenden neben der Fachkompetenz auch Methoden-, Sozial-, und Personalkompetenz (Markowitsch, Messerer \& Prokopp, 2004, S. 79).

Hürden bei der Umsetzung der Projektmethode an der Hochschule können der hohe Arbeitsaufwand beim Planen und Durchführen sowie eventuell notwendige materielle und finanzielle Ressourcen sein. Des Weiteren können bürokratische und organisatorische Hindernisse, wie z. B. Prüfungsordnungen und Stundenpläne der Studierenden, die Durchführung eines Projektes während des laufenden Semesters erschweren. Kritiker dieser Methode merken an, dass die systematische und methodisch fundierte Vermittlung von Wissen und Inhalten auf der Strecke bleiben kann und nicht alle später relevanten Inhalte abgedeckt werden (Markowitsch et al., 2004, S. 79).

Bei dem hier skizzierten Konzept lassen sich solche Risiken bestmöglich abwenden. Dies geschieht, indem

- vor der Arbeit in den Projektgruppen gemeinsame Sitzungen stattfinden, in denen die Grundlagen gemeinsam erarbeitet werden,

- regelmäßig ein gemeinsamer Austausch des gesamten Seminars stattfindet,

- die Aufgaben innerhalb der Projektgruppen konkret formuliert werden und

- die Ideen zur Problemlösung wissenschaftlich fundiert belegt werden.

Zugleich genügt es, manche Aspekte exemplarisch in der Tiefe zu studieren und sich über die anderen einen Überblick zu verschaffen, da es ohnehin nicht möglich ist, alle Details des breiten Berufsfeldes, in dem sie als Lehrende tätig sein werden, zu studieren (Kuhlmeier \& Uhe, 1998, S. 124). 


\section{Marketing im Gastgewerbe |}

\section{Fazit und Ausblick}

Der Artikel hat aufgezeigt, dass die Inhalte des Seminars Grundlagen des Marketings viele Schnittmengen mit denen der Lehrpläne für das Gastgewerbe in NRW aufweisen. Diese finden sich allerdings nicht in einem Lernfeld der Ausbildung, sondern sind mehreren zugeordnet, so dass Kompetenzen im Bereich des Marketings in der gesamten gastgewerblichen Ausbildung relevant sind. Die Fachwissenschaft hat somit für den vorgestellten Fall einen elementaren Stellenwert im zukünftigen Handlungsfeld der Studierenden. Allerdings ist die Entwicklung von Modulen bzw. Seminaren passgenau zu einzelnen Lernfeldern in einer sinnvollen Weise nicht möglich und aufgrund der Exemplarität auch nicht nötig. Alleine die Vielzahl und Heterogenität der Ausbildungsberufe des Berufsfeldes lässt dies in einer solchen Exaktheit nicht zu (Sloane \& Krakau, 2010, S. 76ff.).

Weiterhin wurde eine Skizze der didaktischen Umsetzung an der Hochschule vorgestellt, die nach dem Prinzip des handlungsorientierten Unterrichts an Berufskollegs organisiert ist. Für die Zukunft steht das Entwerfen eines seminarübergreifenden Projektes als weitere Realisierungsmöglichkeit im Fokus. Dabei arbeiten Seminare eines Moduls oder der Fachdidaktik und Fachwissenschaft zusammen.

\section{Literatur}

Bader, R. (2004). Handlungsorientierung als didaktisch-methodisches Konzept der Berufsbildung. In M. Müller \& R. Bader (Hrsg.), Unterrichtsgestaltung nach dem Lernfeldkonzept (S. 61-68). Bielefeld: Bertelsmann.

Bader, R., Schröder, B. \& Gebert, A. (2010). Hochschulorte und Studierendenzahlen in den beruflichen Fachrichtungen. In J.-P. Pahl (Hrsg.), Handbuch berufliche Fachrichtungen (S. 208-222). Bielefeld: Bertelsmann.

Bundesinstitut für Berufsbildung. (2015). „Datenbank Auszubildende“ des Bundesinstituts für Berufsbildung (BIBB) auf Basis der Daten der Berufsbildungsstatistik der statistischen Ämter des Bundes und der Länder (Erhebung zum 31. Dezember)

[https://www2.bibb.de/bibbtools/de/ss1/1865.php].

Brinker, T. \& Schumacher, E.-M. (2014). Befähigen statt belehren: Neue Lehr- und Lernkultur an Hochschulen. Bern: hep.

Deutscher Hotel- und Gaststättenverband. (2015). Ausbildung garantiert Zukunft [www.dehoga-bundesverband.de/ausbildung-karriere/ausbildungsberufe/].

Ertel, H. \& Wehr, S. (2007). Bolognagerechter Hochschulunterricht. Herausforderungen durch Kompetenzorientierung und Lernerzentrierung. In S. Wehr (Hrsg.), Aufbruch in der Hochschullehre. Kompetenzen und Lernende im Zentrum; Bei- 


\section{Marketing im Gastgewerbe}

träge aus der hochschuldidaktischen Praxis (S. 13-28). Bern, Stuttgart, Wien: Haupt.

Fegebank, B. (2010). Berufliche Fachrichtung Ernährung und Hauswirtschaft. In J.P. Pahl \& V. Herkner (Hrsg.), Handbuch Berufliche Fachrichtung (S. 575-587). Bielefeld: Bertelsmann.

Friese, M. (2010). Didaktisch-curriculare Aspekte für Fachrichtungen und Fachrichtungsbereiche personenbezogener Dienstleistungsberufe. In J.-P. Pahl \& V. Herkner (Hrsg.), Handbuch Berufliche Fachrichtung (S. 311-327). Bielefeld: Bertelsmann.

Gudjons, H. (2003). Didaktik zum Anfassen: Lehrer/in-Persönlichkeit und lebendiger Unterricht. Bad Heilbrunn/Obb.: Julius Klinkhardt.

Jung, H. (2010). Allgemeine Betriebswirtschaftslehre. München: Oldenbourg. [http://dx.doi.org/10.1524/9783486719055].

Kettschau, I. (2013). Berufsfeld Ernährung und Hauswirtschaft: Heterogenität als Merkmal - Gemeinsamkeit als Chance. Haushalt in Bildung \& Forschung, 1, 3-15.

Kettschau, I. \& Wirth, K. (2015). Merkmale von Auszubildenden und Ausbildungserfolg an ausgewählten Berufen des Berufsfeldes Ernährung und Hauswirtschaft; Deutschland 2012 (Seminarunterlagen). Münster: Institut für Berufliche Lehrerbildung.

KMK-Sekretariat der Kultusministerkonferenz. (2011). Handreichung für die Erarbeitung von Rahmenlehrplänen der Kultusministerkonferenz für den berufsbezogenen Unterricht in der Berufsschule und ihre Abstimmung mit Ausbildungsordnungen des Bundes für anerkannte Ausbildungsberufe. Bonn.

Kuhlmeier, W. \& Uhe, E.(1998). Fachdidaktik Bau-, Holz- und Gestaltungstechnik. In B. Bonz \& B. Ott (Hrsg.), Fachdidaktik des beruflichen Lernens (S. 103-133). Stuttgart: Franz Steiner.

Markowitsch, J., Messerer, K. \& Prokopp, M. (2004). Handbuch praxisorientierter Hochschulbildung. Wien: facultas wuv universitätsverlag.

Meffert, H. \& Bruhn, M. (2006). Dienstleistungsmarketing: Grundlagen - Konzepte - Methoden. Wiesbaden: Springer.

Meffert, H., Burmann, C. \& Kirchgeorg, M. (2015). Marketing: Grundlagen marktorientierter Unternehmensführung Konzepte - Instrumente - Praxisbeispiele. Wiesbaden: Springer. [http://dx.doi.org/10.1007/978-3-658-02344-7].

Mietzel, G. (2007). Pädagogische Psychologie des Lernens und Lehrens. Göttingen: Hogrefe.

Ministerium für Schule, Jugend und Kinder des Landes Nordrhein-Westfalen. (2004a). Lehrplan für das Berufskolleg in Nordrhein-Westfalen: Ausbildungsberufe im Gastgewerbe. Frechen: Ritterbach. 


\section{Marketing im Gastgewerbe |}

Ministerium für Schule, Jugend und Kinder des Landes Nordrhein-Westfalen. (2004b). Lehrplan für das Berufskolleg in Nordrhein-Westfalen: Köchin/Koch. Frechen: Ritterbach.

Schelten, A. (2010). Einführung in die Berufspädagogik. Stuttgart: Franz Steiner.

Seidel, T. \& Krapp, A. (Hrsg.). (2014). Pädagogische Psychologie: Mit OnlineMaterialien zum Download. Weinheim/Bergstraße: Beltz.

Sloane, P. F. E. \& Krakau, U. (2010). Zum Problemzusammenhang von Beruflichen Fachrichtungen und Berufsfeldern. In J.-P. Pahl \& V. Herkner (Hrsg.), Handbuch Berufliche Fachrichtung (S. 74-87). Bielefeld: Bertelsmann.

Stomporowski, S. (2011). Handlungsfelder der Fachdidaktik Ernährungs- und Haushaltswissenschaften. In S. Stomporowski (Hrsg.), Die Vitamine liegen unter der Schale: Beiträge zur Didaktik der Ernährungs- und Haushaltswissenschaften (S. 7-25). Baltmannsweiler: Schneider Verlag Hohengehren.

Universität Paderborn \& Hochschule Ostwestfalen-Lippe (Hrsg.). (2015). Lehramt an Berufskollegs mit den beruflichen Fachrichtungen Ernährungs- und Hauswirtschaftswissenschaft sowie Lebensmitteltechnik (Kooperationsstudiengang mit der Hochschule Ostwestfalen-Lippe): Prüfungsordnungen.

[http://plaz.uni-paderborn.de/lehrerbildung/lehramtsstudium-undpruefungen/lehramtsstudium-bachelor-of-education/bed-bk-lebensmitteltechnikund-hauswirtschaftswissenschaft/].

\section{Verfasserinnen}

Nicole Fritsche, M.Sc. und Julia Hirsch, M.Sc.

Universität Paderborn - Institut für Ernährung, Konsum und Gesundheit

Warburger Straße 100

D-33098 Paderborn

E-Mail: Nicole.Fritsche@uni-paderborn.de

Julia.Hirsch@uni-paderborn.de 